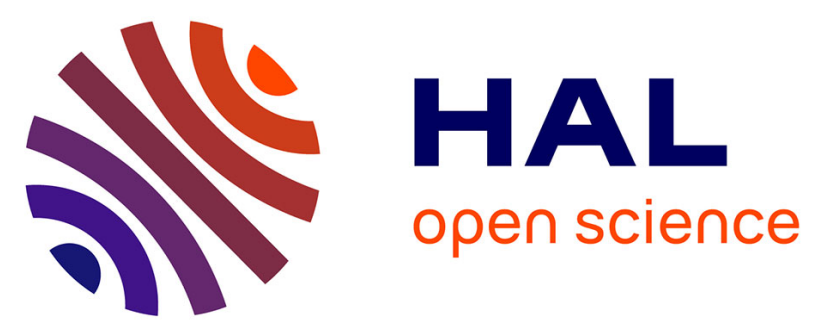

\title{
Humoral and cellular immune correlates of protection against bubonic plague by a live Yersinia pseudotuberculosis vaccine
}

Christian E. Demeure, Anne Derbise, Chloé Guillas, Christiane Gerke, Simon Cauchemez, Elisabeth Carniel, Javier Pizarro-Cerdá

\section{To cite this version:}

Christian E. Demeure, Anne Derbise, Chloé Guillas, Christiane Gerke, Simon Cauchemez, et al.. Humoral and cellular immune correlates of protection against bubonic plague by a live Yersinia pseudotuberculosis vaccine. Vaccine, 2019, 37 (1), pp.123-129. 10.1016/j.vaccine.2018.11.022 . pasteur02067807

\section{HAL Id: pasteur-02067807}

\section{https://hal-pasteur.archives-ouvertes.fr/pasteur-02067807}

Submitted on 14 Mar 2019

HAL is a multi-disciplinary open access archive for the deposit and dissemination of scientific research documents, whether they are published or not. The documents may come from teaching and research institutions in France or abroad, or from public or private research centers.
L'archive ouverte pluridisciplinaire HAL, est destinée au dépôt et à la diffusion de documents scientifiques de niveau recherche, publiés ou non, émanant des établissements d'enseignement et de recherche français ou étrangers, des laboratoires publics ou privés. 
1 Humoral and cellular immune correlates of protection against bubonic plague by a

2 live Yersinia pseudotuberculosis vaccine

3 Christian E. Demeure ${ }^{1 \#}$, Anne Derbise ${ }^{1}$, Chloé Guillas ${ }^{1}$, Christiane Gerke ${ }^{2}$, Simon

4 Cauchemez ${ }^{3,4,5}$, Elisabeth Carniel ${ }^{1, *}$ and Javier Pizarro-Cerdá ${ }^{1}$.

5 Christian E. Demeure: cdemeure@pasteur.fr

$6 \quad$ Anne Derbise: anne.derbise@pasteur.fr

$7 \quad$ Chloé Guillas: chloe.guillas@pasteur.fr

$8 \quad$ Christiane Gerke : christiane.gerke@pasteur.fr

9 Simon Cauchemez: simon.cauchemez@pasteur.fr

10 Elisabeth Carniel: elisabeth.carniel@pasteur.fr Javier Pizarro Cerda: javier.pizarro-cerda@pasteur.fr

$13{ }^{1}$ Unité de Recherche Yersinia. Institut Pasteur, 25-28 rue du Dr Roux, 75724 Paris Cedex $14 \quad$ 15, France.

2 Vaccine Programs, Institut Pasteur, 25-28 rue du Dr Roux, 75724 Paris Cedex 15, France.

${ }^{3}$ Unité de Modélisation Mathématique des Maladies Infectieuses, Institut Pasteur, 25-28 rue du Dr Roux, 75724 Paris Cedex 15, France.

$19{ }^{4}$ Centre National de la Recherche Scientifique, URA3012, 25-28 rue du Dr Roux, 75724

20 Paris Cedex 15, France

$21{ }^{5}$ Center of Bioinformatics, Biostatistics and Integrative Biology, 25-28 rue du Dr Roux, 75724 Paris Cedex 15, France

*Present address: Pasteur Center of Cameroon, BP 1274, Yaounde, Cameroon.

Short title: Correlates of protection for a live plague vaccine

\#: Corresponding author: Dr C.E. Demeure,

$$
\text { Fax: (33-1)-40-61-30-01. }
$$




\section{Highlights:}

32

- IgG \& cells triggered by a $Y$. pseudotuberculosis live vaccine each protect against 34 bubonic plague

- IFNy production and IgG to F1, Yops or sonicated Y. pestis correlate to each other and 36 to protection

- Anti-F1 lgG is the easiest and statistically best predictor of protection, transposable to humans

39 
Abstract:

Immunization with the live-attenuated Yersinia pseudotuberculosis VTnF1 strain producing a Yersinia pestis $\mathrm{F} 1$ pseudocapsule efficiently protects mice against bubonic and pneumonic plague. In clinical trials, demonstration of a plague vaccine's efficacy in humans will not be feasible, and correlates of protection will be needed to bridge the immune response of protected animals to that of vaccinated humans. Using serum transfer and vaccination of antibody-deficient $\mu \mathrm{MT}$ mice, we established that both humoral and cellular responses elicited by VTnF1 independently conferred protection against bubonic plague. Thus, correlates were searched for in both responses, using blood only. Mice were vaccinated with increasing doses of $\mathrm{VTnF} 1$ to provide a range of immune responses and survival outcomes. The cellular response was evaluated using an in vitro IFNy release assay, and IFNy levels were significantly associated with protection, although some survivors were negative for IFNy, so that IFNy release is not a fully satisfactory correlate. Abundant serum IgG against the F1 capsule, Yop injectable toxins, and also non-F1 Y. pestis antigens were found, but none against the LcrV antigen. All readouts correlated to survival and to each other, confirming that vaccination triggered multiple protective mechanisms developing in parallel. Anti-F1 lgG was the most stringent correlate of protection, in both inbred BALB/c mice and outbred OF1 mice. This indicates that antibodies $(\mathrm{Ab})$ to $\mathrm{F} 1$ play a dominant role for protection even in the presence of $\mathrm{Ab}$ to many other targets. Easy to measure, the anti-F1 IgG titer will be useful to evaluate the immune response in other animal species and in clinical trials.

\section{Keywords:}

Plague, live vaccine, Yersinia pestis, Yersinia pseudotuberculosis, correlates of protection, $F 1, \lg G$ 


\section{Introduction}

Plague is caused by $Y$. pestis, one of the deadliest infectious agents afflicting humans. In nature, Y. pestis is mainly a zoonotic pathogen, infecting rodents in large endemic territories throughout the world. Therefore, plague cannot be eradicated and has a permanent capacity to pass to humans. Despite the availability of antibiotic treatments, plague still has a high level of mortality, due mainly to the fast development of pathogenesis. In addition, $Y$. pestis strains showing resistance to several antibiotics have emerged [1, 2] and $Y$. pestis is a potential bioweapon [3]. To face this threat, the development of safe and effective vaccination strategies is critical.

We previously reported that $Y$. pseudotuberculosis can be used as vaccine against plague [4] because the two species share high-level genetic and antigenic identity, including the same type III secretion system and injectable Yop toxins (Yersinia outer proteins [5]). We constructed the Y.pseudotuberculosis strain VTnF1, highly and irreversibly attenuated by deletion of three essential virulence factors (High Pathogenicity Island, pH6/PsaA antigen and YopK toxin). In addition, insertion of the caf operon into the chromosome allows production of a Y.pestis F1 pseudocapsule [4]. A single oral vaccination with live VTnF1 confers protection against bubonic and pneumonic plague in mice [4].

The licensure of a human vaccine requires evaluation of its efficacy in humans. For plague, this is problematic because cases are too sporadic in the world for a field evaluation and human challenge studies are not ethical. In such circumstances, demonstrating efficacy in a well-understood animal model that resembles disease in humans can be considered for approval under the "animal rule" in the USA [6, 7], or the "Extraordinary Use New Drugs Pathway" in Canada [8]. In the EU, a recent perspective from the EMA stated that this does not preclude 'the possibility that animal models data in principle could have a critical role in the assessment' [9]. 
To bridge protection in animals to parameters evaluated during the clinical trials in

94 humans, the investigational compound's mechanism of action to prevent the toxic effects of the pathogen need to be well understood. For vaccines, it should lead to the identification of a relevant biomarker/correlate of protection in animals, presumed to also correlate with protection in humans. Also, identifying correlates of protection for vaccine-induced immunity is crucial during vaccine development [10], e.g. as it enables consistency assessment of vaccine production for complex vaccines by identifying the essential antigen(s) for quantification, and can be used for follow up of populations after immunization, including to determine the need and/or frequency of boosters.

When modified or killed $Y$. pestis have been used as vaccine, both antibodies (Ab) and the cellular arm of immunity contributed to protection [11-13]. The recently developed F1-V recombinant vaccines are based on a combination of F1 and LcrV antigens and induced high levels of $A b$ against these targets in mice or non-human primates (NHP). Ab against these antigens are protective [14-16] and their level predicts protection [15, 17]. Whereas measuring $\mathrm{Ab}$ as correlate for a vaccine consisting of two purified antigens seems straightforward, the definition of a correlate is more complicated for live vaccines due to their complex composition and the humoral and cellular immune mechanisms triggered.

110 Correlates must be relatively easy to measure, so serum antibodies have caught most of

111 the attention. However, the $\mathrm{T}$ cell response may also contribute to protective immunity 112 against $Y$. pestis [11]. As compared to assays measuring antibodies, those measuring 113 cellular responses are more challenging due to logistic and technical limitations [18]. They 114 include the complexity of the assays, the fragility and diversity of living cells, and the 115 absence of standardization of $\mathrm{T}$ cell functions. For any test, the biomarker must be 116 measured using the only biological material safely available in humans: the blood.

117 In this study, we examined which humoral and/or cellular immunological readout 118 correlated with protection in mice vaccinated with the VTnF1 strain, in order to determine 
parameters that can be further developed for evaluation in other animal models and use in

120 future human trials.

121

\section{Materials and Methods}

123

124

125

\section{Bacterial strains}

Y. pestis and Y.pseudotuberculosis isolates and their derivatives have previously been described $[4,19]$.

\section{Animal vaccination and infection}

Institut Pasteur animal facilities are accredited by the French Ministry of Agriculture ( $N^{\circ} B 75$ 15-01; May 22 ${ }^{\text {nd }}, 2008$ ), in compliance with the French and European regulations on care and protection of the Laboratory Animals (EC Directive 86/609, French Law 2001486; June 6, 2001). The research protocol was approved by the Institut Pasteur Ethics Committee for Animal Experimentation and the French Ministry of Research ( $\mathrm{N}^{\circ} 2013-$ 0038). Seven-week-old female OF1, BALB/c and C57BL/6 (B6) mice were from Charles River France. $\mu \mathrm{MT}\left(\mathrm{C} 57 \mathrm{BL} / 6 \mu \mathrm{mt}^{-/}\right)$mice [20] were bred in the Institute. Vaccination consisted in a single dose of VTnF1 $(200 \mu \mathrm{l})$ inoculated intragastrically (ig). Blood was collected 3 weeks after vaccination to obtain immune serum (IS).

Infections with $Y$. pestis were performed in a BSL3 animal facility as previously [4, 19]. Y. pestis CO92 grown at $28^{\circ} \mathrm{C}$ was re-suspended in saline and bubonic plague was induced by injection of $10^{3} \mathrm{CFU}$ (i.e. $10^{2} \times \mathrm{LD}_{50}$, in $50 \mu \mathrm{l}$ ) subcutaneously (sc) in ventral skin. Animals clinical state and survival was monitored daily for 21 days.

\section{Immuno-assays}

$\operatorname{lgG}$ specific for Yersinia were quantified by ELISA as described previously [21]: microtiter plates (NUNC) were coated with either purified $\mathrm{F} 1$ antigen, or sonicated $Y$. pestis CO92 $\Delta$ caf (containing all $Y$. pestis antigens except F1 [4]), or purified recombinant LcrV antigen, or purified Yops, all at $5 \mu \mathrm{g} / \mathrm{ml}$ in PBS, except LcrV: $1 \mu \mathrm{g} / \mathrm{ml}$. The ELISA using Yops was initially developed for the sero-diagnostic of infection by pathogenic Yersiniae 
146 [22]. The Yops solution used contains a mix of all the Yersinia Outer Proteins (Yops)

147 produced in medium by bacteria grown at $37^{\circ} \mathrm{C}$ in the absence of Calcium, as described by

148 Michiels [23]. Sera diluted in PBS containing 0.1\% BSA were incubated in coated plates.

149 Bound IgG were detected by a mouse IgG-specific, HRPO-coupled, rat antibody (Bio-Rad.

150 Antibody titers were calculated as the reciprocal of the lowest sample dilution giving a

151 signal equal to two times the background.

152 Cell-mediated response of vaccinated animals

153 Blood collected on heparin $(50 \mathrm{U})$ was centrifuged on a Lympholyte®-Mammal 154 gradient (Cedarlane, manufacturer' protocol) to separate leukocytes from plasma and 155 erythrocytes. Plasma was frozen for $\lg G$ evaluation, while leukocytes were re-suspended in 156 culture medium: RPMI $1640+$ Glutamax $^{T M}$ (Invitrogen) containing 10\% bovine serum

157 (Lonza), Penicillin /Streptomycin (Life Sciences) and $10 \mathrm{mM}$ B-mercapto-ethanol. 158 Leukocytes $\left(10^{6}\right.$ cells) were restimulated in vitro using either sterile F1 antigen or sonicated $159 Y$. pestis CO92 $\Delta$ caf (both at $5 \mu \mathrm{g} / \mathrm{ml}$ ). Supernatants were collected after $48 \mathrm{~h}$ for cytokine 160 measurements (IFNy and IL-17 Duosets, R\&D Systems).

Transfer of immune serum and vaccination of antibody-deficient $\mu \mathrm{MT}$ mice

162 To evaluate the protective value of antibodies, OF1 mice received either normal or 163 immune serum $(500 \mu \mathrm{l})$ intraperitoneally (ip) two hours before a bubonic plague challenge

164 (CO92, $10^{3} \mathrm{CFU}$ sc). To evaluate the protective value of the cellular immune response, $165 \mu \mathrm{MT}$ mice (unable to produce antibodies [20]) and immune-competent B6 littermates were 166 vaccinated. Because VTnF1 was harmful for immuno-deficient $\mu \mathrm{MT}$ mice (Fig S1), 167 vaccinated and control mice received immune serum (100 $\mu \mathrm{lip})$ at the time of vaccination, 168 according to Parent et al. [12]. Specific antibodies in blood were evaluated by ELISA every

169 four weeks, and mice were challenged when antibodies were undetectable in $\mu \mathrm{MT}$ mice.

\section{Statistical analysis}

171 We studied the association between $\lg$ titers $\left(\log _{10}\right.$ transformed) and the probability 172 to survive a plague challenge. Univariate logistic regressions were used to compute the 
173 odds ratio for survival to the plague challenge for each 10 -fold increase in lgG titers ( $R$ software

174 3.3.2). The Akaike's Information Criterion (AIC) was calculated to compare logistic 175 regression models with the best fitting model, having the lowest AIC. The significance level 176 chosen for all analysis was $p<0.05$ (two-tailed). The Prism software (GraphPad) was used 177 for other statistics: the Fisher exact test to compare survival rates, the unpaired, two-tailed

178 Mann-Whitney test to compare groups for antibody titers and cytokines levels, and the two179 tailed Spearman's rank test for correlation analyses.

180

\section{$181 \quad$ RESULTS}

VTnF1 vaccine-induced protection is mediated by both humoral and cellular immunity

We previously reported that oral vaccination with the VTnF1 strain protected against bubonic plague and induced the production of both specific antibodies and memory cells [4]. To determine which immunological effectors conferred protection, the contribution of antibodies was examined by passive serum transfer. Serum from VTnF1-vaccinated mice or naïve mice (pools from 14 mice) was injected to naïve mice 2 hours prior to infection. Whereas mice with non-immune serum or no serum died, those with immune serum survived (Fig. 1A), showing that VTnF1-specific antibodies alone protected against plague.

191 To evaluate the importance of cellular immunity, $\mu \mathrm{MT}$ mice (unable to produce antibodies) were vaccinated. Because these mice are too immuno-compromised to tolerate the live

193 vaccine (Fig.S1), immune serum was injected to $\mu$ MT mice and B6 littermates at the time of

194 vaccination [12], allowing $100 \%$ survival. When IgG were no longer detected in blood of $195 \mu \mathrm{MT}$ mice (> 2 months), animals were challenged with $Y$. pestis. All vaccinated $\mu \mathrm{MT}$ and B6 196 mice survived, whereas unvaccinated mice (having received and eliminated the antibodies) 197 died (Fig.1B), showing that VTnF1-induced cell-mediated immunity alone protected against 198 plague. 


\section{Evaluation of humoral parameters as correlates of protection against plague}

201

202

203

204

205

206

207

208

209

210

211

212

213

214

215

216

217

218

219

220

221

222

223

224

225

To mimic the heterogeneity of a vaccinated population, groups of mice were vaccinated with suboptimal (no vaccine, $10^{6} \mathrm{CFU}, 10^{7} \mathrm{CFU}$ ) to optimal $\left(10^{8} \mathrm{CFU}\right)$ doses. A dosedependent acquisition of protection was observed in both outbred (OF1) and inbred BALB/c or C57BL/6 (B6) mice (Table 1).

To fit with immunogenicity assays in clinical trials, all immune response analyses were performed with blood, the simplest sample for analysis of the human response. Serum IgG against $F 1$, Yops and a preparation of $Y$. pestis antigens other than $F 1$ were quantified. $F 1$

is specific for Y. pestis, whereas LcrV and Yops are common to pathogenic Yersiniae. Both are protective targets abundant in vivo $[24,25]$. IgG against $\mathrm{F} 1$, Yops and non-F1 Y. pestis antigens could be evidenced (Fig. 2), but no anti-LcrV IgG above the $50 \mathrm{pg} / \mathrm{ml}$ limit of detection of the ELISA, determined using a monoclonal Ab (not shown). Comparable results were obtained with inbred BALB/c or outbred OF1 mice. Despite intra-group variability of IgG levels in all groups, the dose of vaccine clearly dictated the magnitude of the antibody response (Fig. 2 A). In addition, IgG levels against a given target significantly correlated with the levels against the other antigens (Fig. 2 B).

\section{Evaluation of vaccine-induced cellular memory against plague}

In vitro stimulated splenocytes from VTnF1 -vaccinated mice produce IFNy, revealing a type 1 cellular response (Th1) [4]. Here, the IFNy release assay was applied to blood leukocytes restimulated with either $\mathrm{F} 1$ or sonicated Y.pestis C092 $\Delta$ caf (all Y.pestis antigens except F1). Purified F1 failed to induce any IFNy production, whereas the sonicated Y. pestis induced IFNy in the majority of samples (Fig. 3A). Unspecific stimulation was unlikely, because cells from naïve mice did not respond. All mice positive for IFNy survived plague, although IFNy was not detected in all protected individuals, yielding a $69 \%$ confidence (18 mice / 26 survivors; Fig. 3A). IFNy correlated significantly with anti-F1 lgG 
226 (Fig. 3B) as well as with anti-Y. pestis $\Delta$ caf IgG (Fig. 3C), and both increased according to 227 the vaccine dose.

IL-17 plays a protective role against plague [26], and is produced by splenocytes from VTnF1-vaccinated mice [4]. However, blood leukocyte cultures yielded only very low amounts (not shown) and thus was not further investigated.

\section{Logistic regression analysis of serological immune readouts}

In BALB/c mice (naïve and vaccinated using all doses), the three serum lgG titers 234 (against F1, Yops \& sonicated Y. pestis) were strongly associated with survival. IgG anti-F1 and IgG anti-Yersinia titers perfectly separated mice that survived and those that died. All mice with $\lg G$ anti-F1 $\leq 10^{3}$ and all those with $\lg G$ anti- Yersinia $\leq 3 \times 10^{2}$ died while all others survived (Fig. 2A \& 4A, Fig. S2A). IgG anti-Yops titers also almost perfectly separated the mice by outcome: most protected mice (28/29) had IgG anti-Yops above 150, and no one which died (Fig. S2B).

240 Because the human population is genetically diverse, the possibility to predict 241 protection was examined in outbred OF1 mice. IgG levels were more heterogeneous (Fig. 242 S3), but each IgG titer was significantly associated with survival as revealed using 243 univariate logistic regressions (Fig. 4B, Fig. S2B,2D), with $p<0.001$ (Table 2). Based on the 244 AIC, the anti-F1 IgG titer had the strongest association with survival to bubonic plague. For 245 each 10-fold increase in anti-F1 IgG titer, the odd ratio for survival to the plague challenge 246 was 3.5 (95\% confidence interval: 2.1, 7.0) (Table 2; Fig. 4B).

\section{Discussion}

Identifying correlates of protection is of high interest during vaccine development, to 251 identify key antigenic components for consistency evaluation of vaccine production, to 
evaluate the responses of individuals and populations after vaccination, and, as in the case

253 for a plague vaccine for which efficacy trials are not ethical, to establish parameters for 254 evaluation [10]. In order to prepare for future evaluation of the immune response in other animal models and particularly in volunteers in clinical trials, the present work aimed at identifying a correlate of protection in mice vaccinated with VTnF1, which could be measured using blood. In addition to measuring an immune parameter with robust statistical correlation to protection, the test had to be as simple as possible to perform.

We found that both humoral and cellular responses elicited by VTnF1 had a protective capacity, so that parameters measuring each could correlate to protection. Among the analyzed readouts, the anti-F1 IgG titer was the most significant predictor, and therefore represented the best correlate of protection of our study. In agreement, the only mouse (1/14 OF1) not protected 6 months after vaccination in a previous study [4] had progressively lost its anti-F1 IgG after vaccination (Fig S4). While it has previously been reported that anti-F1 Ab were good correlates of protection in mice and NHP, for F1 + LcrV vaccines [17], F1-V fusion protein [16, 27, 28], or F1-producing live Salmonella or Poxvirus vectors [29, 30], our results highlight the fact that $A b$ against $F 1$ play a dominant role for protection even in the presence of Ab to many other targets. This supports the conclusion that $\mathrm{F} 1$ is a major target for protective immunity. The function of these anti-F1 antibodies against $Y$. pestis remains to be evaluated. Because F1 is the principal component of the

271 Y. pestis pseudocapsule, easily accessible, Ab may opsonize and aggregate bacteria,

272 facilitating engulfment and destruction by phagocytes. Such functions may guide the 273 development of functional assays [31].

274 In addition to anti-F1 Ab, our study also shows a significant association between 275 survival and serum IgG Ab directed to Yops or to Yersinia antigens (Y. pestis sonicate, 276 including Yops but not F1). These Ab therefore are likely to contribute to protection. 277 Importantly, immunization with VTnF1 protected mice also against F1-negative plague 278 strains [4]. While in the present study, challenge experiments were performed with the F1- 
positive $Y$. pestis strain, Ab responses against Yops or Yersinia antigens might be essential 280 for protection against F1-negative $Y$. pestis strains and will be subject to future evaluations.

281 No significant serum IgG against LcrV could be observed in VTnF1-vaccinated mice. 282 While it is known that $\mathrm{V}$ is immunogenic when given with adjuvant [32, 33], and is produced 283 by our vaccine strain, our finding is in agreement with previous reports that almost no $A b$ 284 against $\mathrm{V}$ were produced by murine or human plague survivors, and only low amounts by mice vaccinated with various attenuated $Y$. pestis strains [32-36], whereas Ab to F1 were abundant [34, 37]. $\mathrm{V}$ produced by live Yersinia strains therefore appears poorly immunogenic. This could results from the ability of $V$ to induce tolerogenic dendritic cells [38], which could prevent the development of V-specific $T$ cells. This function could be active in live strains, but overcome by the adjuvant's effects in molecular vaccines such as 290 F1V.

Besides the humoral response, cellular immune responses alone were also sufficient for protection as demonstrated by the survival of $\mu \mathrm{MT}$ mice lacking Ab (the present work and $[12,13])$. This was in line with the expectation that in contrast to molecular vaccines which principally prime humoral immunity and type $2 \mathrm{~T}$ cells [39, 40], vaccines comprised of replicating agents (live attenuated organisms, for example BCG) [41]. Also, it has been shown that $A b$ and $T$ cells collaborate to protect against $Y$. pestis $[12,13]$. IFNY, released by type $1 \mathrm{~T}$ cells, activates macrophages and dendritic cells against bacteria, and its injection to mice during plague induced survival [42]. Therefore, an IFNY-release assay could have predicted protection against plague. The cellular response induced by VTnF1 indeed involved IFNy production and thus included Th1 cells. However, IFNy was detected

301 in most protected individuals, but not in all of them. IFNy levels and anti-F1 IgG were 302 correlated, indicating that humoral and cellular mechanisms developed in parallel. Thus, 303 IFNy release is a statistically significant but not fully satisfying correlate of protection.

F1 triggered a very low IFNy production by blood leukocytes. In a previous study, 305 splenocytes produced 30 times less IFNy in response to $\mathrm{F} 1$ than to sonicated $Y$. pestis, 
composed of multiple targets [4]. In agreement, plague patients have a low frequency of F1reactive T cells [37]. Furthermore, despite the contribution of IL-17 to plague survival in mice [26], IL-17 was hardly detectable in the blood cultures, probably due to a low Th17 frequency. Thus, these parameters could not be established as correlate of protection.

Live vaccines are known to induce strong humoral and type 1 cellular immunity [41], with the counterpart that they should not be given to people who are immunosuppressed 312 (either due to drug treatment or underlying illness). Public health institutions and 313 professionals are conscientious about this $[43,44]$. Because our vaccine strain is not well 314 tolerated by immunodepressed mice, we currently characterize a new, completely avirulent sub-strain derived from VTnF1, which does not present this risk. The promising results will be the topic of a forthcoming publication.

A future step in the development of our vaccine strains will be the evaluation in 318 primates. In the past, the F1V vaccine failed to protect African Green Monkeys in spite of 319 high anti-F1 antibody titers [45], and this was ascribed to the presence of non-protective 320 antibodies [27]. Whether these antibodies will also be seen in primates vaccinated with 321 VTnF1 will be important to determine, because it is not known whether non-protective 322 antibodies appear to the same extend when antigens are part of a live bacteria or injected 323 in a pure form associated with an adjuvant. In addition, care must be taken not to over324 interpret results obtained in the mouse, because various species previously tested (mice, 325 guinea pigs, primates) have shown great differences in their susceptibility to attenuated 326 strains vaccines and in their responsiveness to vaccines and given antigens [46-48]. In 327 primates and humans, IFNy should also be measured in addition to antibodies to evaluate 328 the role of cellular immunity in protection and to determine which correlate is the best.

In conclusion, our results show that measurement of anti-F1 IgG titer is a correlate of 330 protection against bubonic plague after immunization with the VTnF1 candidate vaccine. A 331 human anti-F1 IgG ELISA test already has been used as a sero-diagnostic tool in plague 332 patients in Madagascar [49]. A similar assay has been used in trials of the F1-V in NHP [50, 
333 51] and a bridge ELISA for anti-F1 Ab established to compare antibody levels in different 334 species was used for evaluation of the $\mathrm{rF} 1 \mathrm{~V}$ vaccine to bridge results obtained in mice, 335 NHP and humans [16, 52]. Because all species do not respond equally to vaccination [53], 336 these tests will have to be adapted and evaluated for further assessment of the VTnF1 337 vaccine in the second animal species tested and in future clinical trials to confirm the value 338 of this correlate in humans.

339 


\section{Acknowledgements}

341 The project was supported by an ANR Emergence grant (ANR-12-EMMA-0011-01) and an

342 Institut Pasteur Accelerating Preclinical Candidates - GPF- Vaccinology 2015 grant 343 (GPFVacc2-08). The authors wish to warmly thank Pierre Goossens for precious advice 344 and support, Henrik Salje for help with statistical analysis, H. Saklani for advice regarding 345 the cell culture protocols and Stéphanie Simon (CEA, Saclay, France) for the kind gift of 346 recombinant LcrV and a mouse monoclonal anti-LcrV.

\section{Conflicts of interests :}

349 Authors declare no conflict of interests.

\section{$351 \quad$ Figure captions}

352 Figure 1: Cellular and humoral immunity independently protect VTnF1-vaccinated mice

353 against plague.

354 A : Groups of seven OF1 mice received either no serum, or serum from normal mice (non355 immune serum), or immune serum obtained from mice vaccinated orally with one dose of 356 VTnF1 (10 $\left.{ }^{8} \mathrm{CFU}\right)$. Immediately after, mice were infected subcutaneously to evaluate 357 resistance to bubonic plague $\left(10^{3}\right.$ Y. pestis CO92 sc). B : Groups of five $\mu \mathrm{MT}$ mice (B cell358 deficient) or wild type C57BL/6 mice were vaccinated or not with VTnF1 (10 $\left.{ }^{8} \mathrm{CFU}\right)$

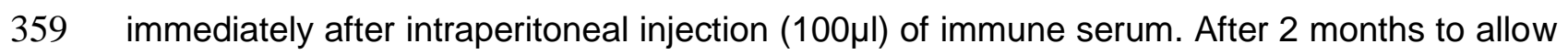
360 clearance of antibodies, mice were infected as in A. Survival significance was evaluated by 361 the Fisher Exact test, using the "no serum" $(A)$ or "no vaccine" $(B)$ condition as reference. 362 ns: not significant, ${ }^{* *}: p<0.01,{ }^{* *}: p<0.0001$

364 Figure 2: Humoral immune response induced by vaccination against different targets.

365 (A) Groups of 14 BALB/c mice were vaccinated orally with one dose of VTnF1 containing $36610^{6}$ (triangles), $10^{7}$ (squares) or $10^{8}$ (circles) CFU, or were not vaccinated (diamonds). 
Blood was taken 3 weeks later and serum IgG specific for sonicated $Y$. pestis $\Delta$ caf 368 antigens, purified F1 or purified Yops were measured by ELISA. Horizontal lines indicate 369 the median value for each dose. Four weeks after vaccination, mice were infected 370 subcutaneously to evaluate resistance to bubonic plague by sc injection of $10^{3} \mathrm{Y}$. pestis 371 CO92. The grey region indicates animals which did not survive. (B) Statistical correlation

372 between IgG levels measured in (A) was determined using Spearman's rank correlation 373 test. The corresponding "rs" coefficient and significance ( $p$ value) are indicated.

375 Figure 3: Cellular immune response of vaccinated mice and its correlation with the humoral 376 response.

377 Groups of 14 BALB/c mice were vaccinated ig with different doses of the VTnF1 strain (10 ${ }^{6}$ : 378 triangles; $10^{7}$ : squares; $10^{8}$ : circles), or were not vaccinated (7 mice: diamonds). Blood was 379 collected 3 weeks after vaccination to collect plasma and perform a leukocytes restimulation 380 assay using a sonicated $Y$. pestis $\Delta$ caf as stimulus. IFNY was measured by ELISA in $48 \mathrm{~h}$ 381 culture supernatants. A: Production of IFNy according to the vaccine dose. The limit of detection 382 (lod) is indicated by a dotted line. B, C: Correlation in the same blood samples between IFNy 383 levels and IgG recognizing $\mathrm{F} 1$ (B) or the sonicated Y. pestis $\Delta$ caf $(\mathrm{C})$, determined as in Fig. 2. 384 Correlations were estimated using Spearman's rank test, which rs coefficient and 385 corresponding $\mathrm{p}$ value are given for each plot. Four weeks after vaccination, mice received an 386 sc injection of $10^{3}$ Y. pestis CO92, and survival was followed 21 days. In all plots, the grey area 387 indicates mice which did not survive the challenge.

389 Figure 4: Survival as a function of anti-F1 lgG titers in BALB/c mice and OF1 mice.

390 Groups of $14 \mathrm{BALB} / \mathrm{c}(\mathrm{A})$ or OF1 (B) mice were vaccinated ig with different doses of the VTnF1 391 strain $\left(10^{6}\right.$ to $10^{8}$ or none, as in Figure 3). Blood was collected 3 weeks after vaccination to

392 collect serum and measure titers of IgG recognizing F1. Four weeks after vaccination, mice 393 received an sc injection of $10^{3}$ Y. pestis CO92, and mice were followed for 21 days. For each 394 individual mouse, the lgG titer is plotted against survival (blue, noted 1) or death (red, noted 0). 
395 For BALB/c mice (A), anti-F1 lgG titers perfectly separated mice who died (IgG anti-F1 396 titers $\leq 10^{3}$ ) from those who survived ( $\lg G$ anti-F1 titers $\left.>10^{3}\right)$ so that the logistic model could 397 not be fitted to the data. For OF1 mice (B), the predicted survival probability obtained with 398 the logistic regression (black line) is plotted along with the 95\% confidence interval (grey 399 area).

\section{Supplemental material}

403 Figure S1: Survival of antibody-deficient $\mu \mathrm{MT}$ mice to vaccination with VTnF1.

$404 \mu \mathrm{MT}$ mice (circles) and C57BL/6 mice (same background lineage as $\mu \mathrm{MT}$; squares)

405 received one oral dose of $\mathrm{VTnF} 1\left(10^{8} \mathrm{CFU}\right.$ ig; 7 mice/ group) and survival was recorded for 40621 days.

408 Figure S2: Survival probability as a function of anti-Yersinia and anti-Yops IgG titers in $409 \quad$ BALB/c and OF1 mice.

410 BALB/c mice (A, B) and OF1 mice (C, D) were vaccinated as in Figure 4, and IgG titers 411 against Yersinia antigens (A, C) and Yops (B, D) were plotted against the survival (blue, 412 noted 1) or death (red, noted 0 ) observed during the plague challenge. For OF1 mice, the 413 predicted survival probability could be calculated by logistic regression (black line) and is 414 plotted along with the 95\% confidence interval (grey area). For BALB/c mice, the titers 415 almost perfectly separated mice that died from those that survived so that the logistic model 416 could not be fitted to the data.

418 Figure S3: Dose-dependent humoral immune response induced by vaccination in outbred 419 OF1 mice 
420 Groups of 14 mice were vaccinated or not with one dose of VTnF1 consisting in $10^{6}, 10^{7}$ or $10^{8}$ 421 CFU ig, and blood was taken 3 weeks later to evaluate seric IgG specific for $Y$. pestis antigens 422 other than F1 or purified F1 or Yops by ELISA. Horizontal lines indicate the median value for 423 each dose of vaccine.

425 Figure S4: Relationship between long-term evolution of the anti-F1 lgG titer and the outcome of 426 a bubonic plague challenge

427 These results were previously reported in [4] and were re-analyzed and plotted here. OF1 428 mice were vaccinated once with $\mathrm{VTnF} 1\left(10^{8} \mathrm{CFU}\right.$ ig) and sera were collected before and 429 every month after up to 6 months. Animals were then infected with Y. pestis (CO92, $10^{3}$ 430 CFU sc) and indicated is the outcome after 3 weeks. Anti-F1 serum IgG titers were 431 determined by Elisa. Shown are individual evolution curves of 14 mice. 
435 [1] Galimand M, Guiyoule A, Gerbaud G, Rasoamanana B, Chanteau S, Carniel E, et al. 436 Multidrug resistance in Yersinia pestis mediated by a transferable plasmid. New England 437 Journal of Medicine. 1997;337:677-80.

438 [2] Guiyoule A, Gerbaud G, Buchrieser C, Galimand M, Rahalison L, Chanteau S, et al. 439 Transferable plasmid-mediated resistance to streptomycin in a clinical isolate of Yersinia 440 pestis. Emerging Infectious Diseases. 2001;7:43-8.

441 [3] Inglesby TV, Dennis DT, Henderson DA, Bartlett JG, Ascher MS, Eitzen E, et al. Plague 442 as a biological weapon - Medical and public health management. Journal of the American 443 Medical Association. 2000;283:2281-90.

444 [4] Derbise A, Hanada Y, Khalife M, Carniel E, Demeure CE. Complete Protection against 445 Pneumonic and Bubonic Plague after a Single Oral Vaccination. PLoS Negl Trop Dis.

$446 \quad 2015 ; 9: \mathrm{e} 0004162$.

447 [5] Cornelis GR. The Yersinia YSC-YOP 'type III' weaponry. Nature Reviews Molecular Cell 448 Biology. 2002;3:742-52.

449 [6] Food Drug Administration CfDER. 21CFR601 - Biologics -Part 601: Licencing. Subpart

450 H: Approval of Biological Products when Human Efficacy Studies are not Ethical or Feasible.

451 In: FDA, editor. FDA Maryland2017.

452 [7] Food Drug Administration CfDER. 21CFR314 - Drugs for Human Use - Part 314:

453 Applications for FDA approval to market a new Drug - Subpart I: Approval of New Drugs

454 when Human Efficacy Studies are not Ethical or Feasible. In: FDA, editor. FDA

455 Maryland2017.

456 [8] Office of Regulatory Affairs. Guidance Document - Submission and Information

457 Requirements for Extraordinary Use New Drugs (EUNDs). In: Health-Canada., editor.

458 Ottawa2014.

459 [9] Cavaleri M, Thomson A, Salmonson T, Hemmings RJ. A viewpoint on European

460 Medicines Agency experience with investigational medicinal products for Ebola. Clinical

461 Trials. 2016;13:101-4.

462 [10] Plotkin SA. Correlates of protection induced by vaccination. Clinical and vaccine 463 immunology : CVI. 2010;17:1055-65.

464 [11] Kummer LW, Szaba FM, Parent MA, Adamovicz JJ, Hill J, Johnson LL, et al.

465 Antibodies and cytokines independently protect against pneumonic plague. Vaccine.

466 2008;26:6901-7.

467 [12] Parent MA, Berggren KN, Kummer LW, Wilhelm LB, Szaba FM, Mullarky IK, et al.

468 Cell-mediated protection against pulmonary Yersinia pestis infection. Infect Immun.

469 2005;73:7304-10.

470 [13] Smiley ST. Current challenges in the development of vaccines for pneumonic plague.

471 Expert review of vaccines. 2008;7:209-21.

472 [14] Zauberman A, Cohen S, Levy Y, Halperin G, Lazar S, Velan B, et al. Neutralization of

473 Yersinia pestis-mediated macrophage cytotoxicity by anti-LcrV antibodies and its correlation

474 with protective immunity in a mouse model of bubonic plague. Vaccine. 2008;26:1616-25.

475 [15] Little SF, Webster WM, Wilhelm H, Fisher D, Norris SL, Powell BS, et al. Quantitative

476 anti-F1 and anti-V IgG ELISAs as serological correlates of protection against plague in

477 female Swiss Webster mice. Vaccine. 2010;28:934-9.

478 [16] Fellows P, Price J, Martin S, Metcalfe K, Krile R, Barnewall R, et al. Characterization of

479 a Cynomolgus Macaque Model of Pneumonic Plague for Evaluation of Vaccine Efficacy.

480 Clinical and vaccine immunology : CVI. 2015;22:1070-8. 
[17] Williamson ED, Vesey PM, Gillhespy KJ, Eley SM, Green M, Titball RW. An IgG1 titre to the $\mathrm{F} 1$ and $\mathrm{V}$ antigens correlates with protection against plague in the mouse model. Clinical and Experimental Immunology. 1999;116:107-14. [18] Salerno-Goncalves R, Sztein MB. Cell-mediated immunity and the challenges for vaccine development. Trends Microbiol. 2006;14:536-42. encapsulated Yersinia pseudotuberculosis is a highly efficient vaccine against pneumonic plague. PLoS NTD. 2012;6:e1528.

[20] Kitamura D, Rajewsky K. Targeted disruption of mu chain membrane exon causes loss of heavy-chain allelic exclusion. Nature. 1992;356:154-6.

[21] Chanteau S, Rahalison L, Ralafiarisoa L, Foulon J, Ratsitorahina M, Ratsifasoamanana $\mathrm{L}$, et al. Development and testing of a rapid diagnostic test for bubonic and pneumonic plague. Lancet. 2003;361:211-6.

[22] Benoit C, Guiyoule A, Carniel E. Sérodiagnostic des infections humaines à Yersinia pathogènes. Presse Méd. 1996;25:1627-30.

[23] Michiels T, Wattiau P, Brasseur R, Ruysschaert JM, Cornelis G. Secretion of Yop Proteins by Yersiniae. Infection and Immunity. 1990;58:2840-9.

[24] Lawton WD, Erdman RL, Surgalla MJ. Biosynthesis and purification of V and W antigens in Pasteurella pestis. Journal of Immunology. 1963;91:179-84.

[25] Flashner Y, Fisher M, Tidhar A, Mechaly A, Gur D, Halperin G, et al. The search for early markers of plague: evidence for accumulation of soluble Yersinia pestis LcrV in bubonic and pneumonic mouse models of disease. FEMS Immunol Med Microbiol. 2010;59:197-206.

[26] Lin JS, Kummer LW, Szaba FM, Smiley ST. IL-17 contributes to cell-mediated defense against pulmonary Yersinia pestis infection. J Immunol. 2011;186:1675-84.

[27] Bashaw J, Norris S, Weeks S, Trevino S, Adamovicz JJ, Welkos S. Development of in vitro correlate assays of immunity to infection with Yersinia pestis. Clinical and vaccine immunology : CVI. 2007;14:605-16.

[28] Williamson ED. Acellular vaccines against plague. In: Carniel E, Hinnebusch B, editors. Yersinia, Systems Biology and Control. Hethersett: Horizon Scientific Press; 2012. p. 123-42. [29] Morton M, Garmory HS, Perkins SD, O'Dowd AM, Griffin KF, Turner AK, et al. A Salmonella enterica serovar Typhi vaccine expressing Yersinia pestis F1 antigen on its surface provides protection against plague in mice. Vaccine. 2004;22:2524-32.

[30] Mencher JS, Smith SR, Powell TD, Stinchcomb DT, Osorio JE, Rocke TE. Protection of black-tailed prairie dogs (Cynomys ludovicianus) against plague after voluntary consumption of baits containing recombinant raccoon poxvirus vaccine. Infect Immun. 2004;72:5502-5. [31] Anderson GW, Worsham PL, Bolt CR, Andrews GP, Welkos SL, Friedlander AM, et al. Protection of mice from fatal bubonic and pneumonic plague by passive immunization with monoclonal antibodies against the F1 protein of Yersinia pestis. American Journal of Tropical Medicine and Hygiene. 1997;56:471-3.

[32] Williamson ED, Eley SM, Griffin KF, Green M, Russell P, Leary SE, et al. A new improved sub-unit vaccine for plague: the basis of protection. FEMS Immunol Med Microbiol. 1995;12:223-30.

[33] Qiu Y, Liu Y, Qi Z, Wang W, Kou Z, Zhang Q, et al. Comparison of immunological responses of plague vaccines $\mathrm{F} 1+\mathrm{rV} 270$ and EV76 in Chinese-origin rhesus macaque, Macaca mulatta. Scand J Immunol. 2010;72:425-33.

[34] Quenee LE, Cornelius CA, Ciletti NA, Elli D, Schneewind O. Yersinia pestis caf1 variants and the limits of plague vaccine protection. Infection and Immunity. 2008;76:202536. 

by Live Yersinia pestis Vaccine. In: V. St. Georgiev KAW, J.J.McGowan, editor. National Institutes of Allergy and Infectious Diseases, NIH Volume 1, Frontiers in Research. Totawa, NJ.: Humana Press; 2008. p. 473-80. [36] Sun W, Sanapala S, Henderson JC, Sam S, Olinzock J, Trent MS, et al. LcrV delivered via type III secretion system of live attenuated Yersinia pseudotuberculosis enhances immunogenicity against pneumonic plague. Infect Immun. 2014;82:4390-404.

[37] Li B, Zhou DS, Wang ZY, Song ZZ, Wang H, Li M, et al. Antibody profiling in plague patients by protein microarray. Microbes and Infection. 2008;10:45-51.

[38] DePaolo RW, Tang FM, Kim I, Han M, Levin N, Ciletti N, et al. Toll-Like Receptor 6 Drives Differentiation of Tolerogenic Dendritic Cells and Contributes to LcrV-Mediated Plague Pathogenesis. Cell Host and Microbe. 2008;4:350-61.

[39] Heath DG, Anderson GW, Mauro JM, Welkos SL, Andrews GP, Adamovicz J, et al. Protection Against Experimental Bubonic and Pneumonic Plague By a Recombinant Capsular F1-V Antigen Fusion Protein Vaccine. Vaccine. 1998;16:1131-7.

[40] Meyer KF, Cavanaugh DC, Bartelloni PJ, Marshall JD, Jr. Plague immunization. I. Past and present trends. Journal of Infectious Diseases. 1974;129:S13-8.

[41] Levine MM, Sztein MB. Vaccine development strategies for improving immunization: the role of modern immunology. Nat Immunol. 2004;5:460-4.

[42] Nakajima R, Brubaker RR. Association between virulence of Yersinia pestis and suppression of gamma interferon and tumor necrosis factor alpha. Infection and Immunity. 1993;61:23-31.

[43] Public Health England. Live attenuated vaccines: avoid use in those who are clinically immunosuppressed. In: GOV.UK, editor. Vigilance, safety alerts and guidance 2016. [44] Lopez A, Mariette X, Bachelez H, Belot A, Bonnotte B, Hachulla E, et al. Vaccination recommendations for the adult immunosuppressed patient: A systematic review and comprehensive field synopsis. J Autoimmun. 2017;80:10-27.

[45] Pitt L. Nonhuman Primates as a Model for Pneumonic Plague. . Public workshop on animal models and correlates of protection for plague vaccines Gaithersburg, Maryland, USA. 2004. [46] Girard G. Immunity in Plague. Acquisitions Supplied by 30 Years of Work on the "Pasteurella Pestis Ev" (Girard and Robic) Strain. Biol Med (Paris). 1963;52:631-731. [47] Hallett AF, Isaacson M, Meyer KF. Pathogenicity and immunogenic efficacy of a live attentuated plaque vaccine in vervet monkeys. Infect Immun. 1973;8:876-81.

[48] Welkos S, Pitt ML, Martinez M, Friedlander A, Vogel P, Tammariello R. Determination of the virulence of the pigmentation-deficient and pigmentation-/plasminogen activatordeficient strains of Yersinia pestis in non-human primate and mouse models of pneumonic plague. Vaccine. 2002;20:2206-14.

[49] Rasoamanana B, Leroy F, Boisier P, Rasolomaharo M, Buchy P, Carniel E, et al. Field evaluation of an immunoglobulin G anti-F1 enzyme-linked immunosorbent assay for serodiagnosis of human plague in Madagascar. Clin Diagn Lab Immunol. 1997;4:587-91. [50] Quenee LE, Ciletti NA, Elli D, Hermanas TM, Schneewind O. Prevention of pneumonic plague in mice, rats, guinea pigs and non-human primates with clinical grade rV10, rV10-2 or F1-V vaccines. Vaccine. 2011;29:6572-83.

[51] Chichester JA, Musiychuk K, Farrance CE, Mett V, Lyons J, Mett V, et al. A single component two-valent LcrV-F1 vaccine protects non-human primates against pneumonic plague. Vaccine. 2009;27:3471-4.

[52] Fellows P, Adamovicz J, Hartings J, Sherwood R, Mega W, Brasel T, et al. Protection in mice passively immunized with serum from cynomolgus macaques and humans vaccinated with recombinant plague vaccine (rF1V). Vaccine. 2010;28:7748-56. 
[53] Feodorova VA, Sayapina LV, Motin VL. Assessment of Live Plague Vaccine Candidates. In: S. T, editor. Vaccine Design Methods in Molecular Biology. New York, NY: Humana Press; 2016.

583 


\section{Percent survival;}

proportion surviving (live/total);

significance

\begin{tabular}{|c|c|c|c|c|}
\hline Mouse lineage & Naives & $10^{6} \mathrm{CFU}$ & $10^{7} \mathrm{CFU}$ & $10^{8} \mathrm{CFU}$ \\
\hline \multirow{3}{*}{ OF1 } & $0 \%$ & $23 \%$ & $67 \%$ & $100 \%$ \\
\hline & $(14 / 14)$ & $(3 / 13)$ & $(14 / 21)$ & $(14 / 14)$ \\
\hline & & ns & $* * *$ & $* * *$ \\
\hline \multirow{3}{*}{ BALB/c } & $0 \%$ & $7 \%$ & $86 \%$ & $100 \%$ \\
\hline & $(0 / 13)$ & $(1 / 14)$ & $(12 / 14)$ & $(13 / 13)$ \\
\hline & & ns & $\star * *$ & $* * *$ \\
\hline \multirow{3}{*}{ C57BL/6 } & $0 \%$ & $\mathrm{nt}$ & $\mathrm{nt}$ & $100 \%$ \\
\hline & $(0 / 12)$ & & & $(12 / 12)$ \\
\hline & & & & $\star \star \star$ \\
\hline
\end{tabular}

Table 1: Protection of various mouse strains against bubonic plague provided by vaccination with graded doses of VTnF1.

OF1 (outbred), C57BL/6 or BALB/c (inbred) mice (groups of 12-21 from 2 experiments) were orally vaccinated with graded doses of $\mathrm{VTnF} 1$ (as indicated), and were exposed four weeks later to bubonic plague (sc injection of $10^{3} Y$. pestis CO92, fatal for naïve mice). Survival was followed for 21 days, and significance of protection was calculated using the Fisher exact test. nt: not tested. ns: not significant, ${ }^{*}: p<0.05,{ }^{\star \star *}: p<0.0001$ against naïve mice. 


\begin{tabular}{lccc} 
& OR $(95 \%$ Cl) & p-value & AIC \\
\hline IgG anti-F1 & $3.5(2.1,7.0)$ & $<0.001$ & 49.4 \\
IgG anti-Yersinia & $7.9(3.1,26.9)$ & $<0.001$ & 54.3 \\
IgG anti-Yops & $7.8(2.9,29.5)$ & $<0.001$ & 58.0 \\
\hline
\end{tabular}

Table 2: Odds ratio for survival to the plague challenge for each 10-fold increase in IgG anti-F1, IgG anti-Yersinia and IgG anti-Yops titers, in OF1 mice.

Odds ratio $(\mathrm{OR})$ and their $95 \%$ confidence intervals $(95 \% \mathrm{Cl})$ were computed with univariate logistic regressions. The Akaike Information Criterion (AIC) is also provided for model comparison. The best fitting model is the one with the smallest AIC. 

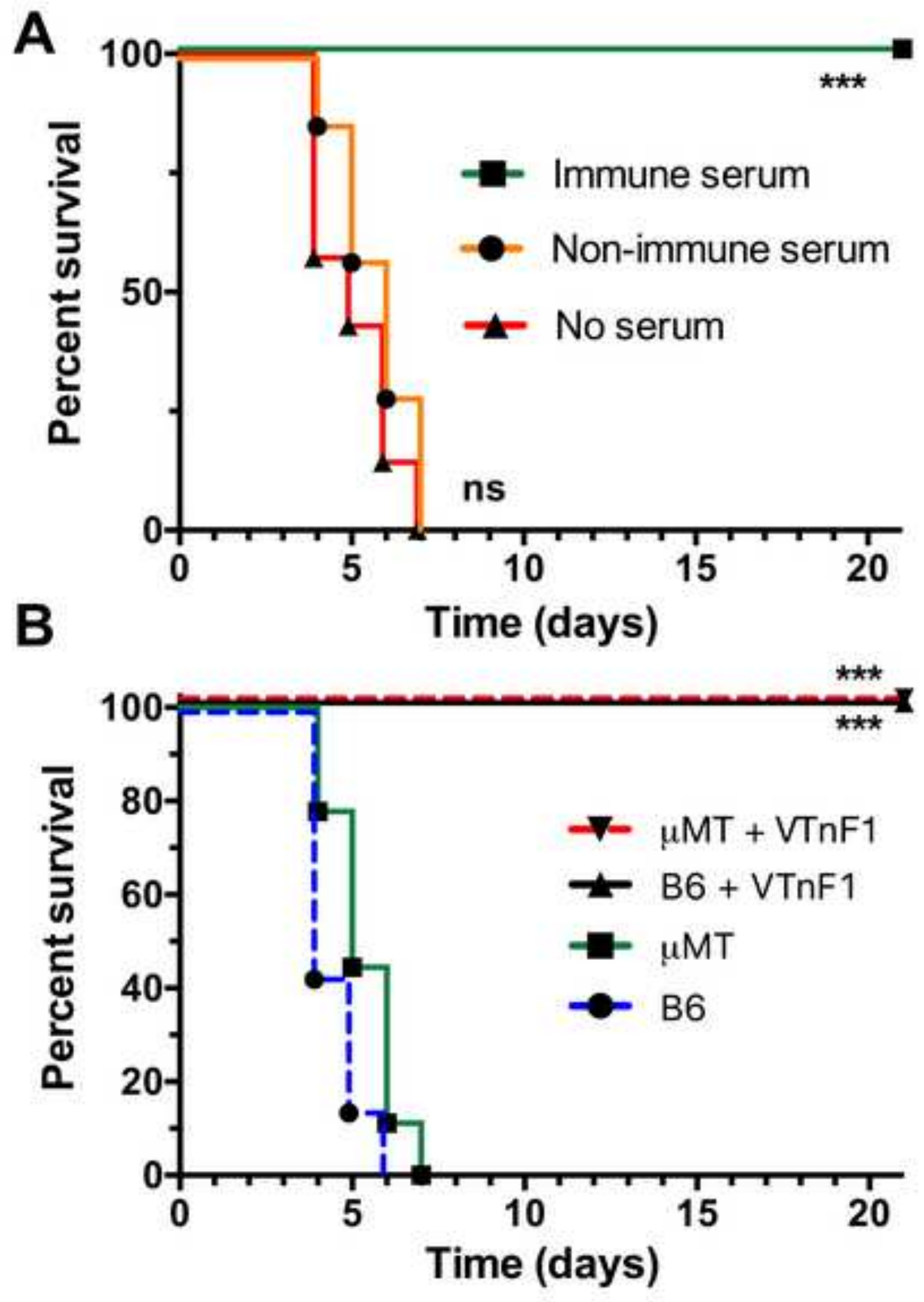


\section{Figure 2}
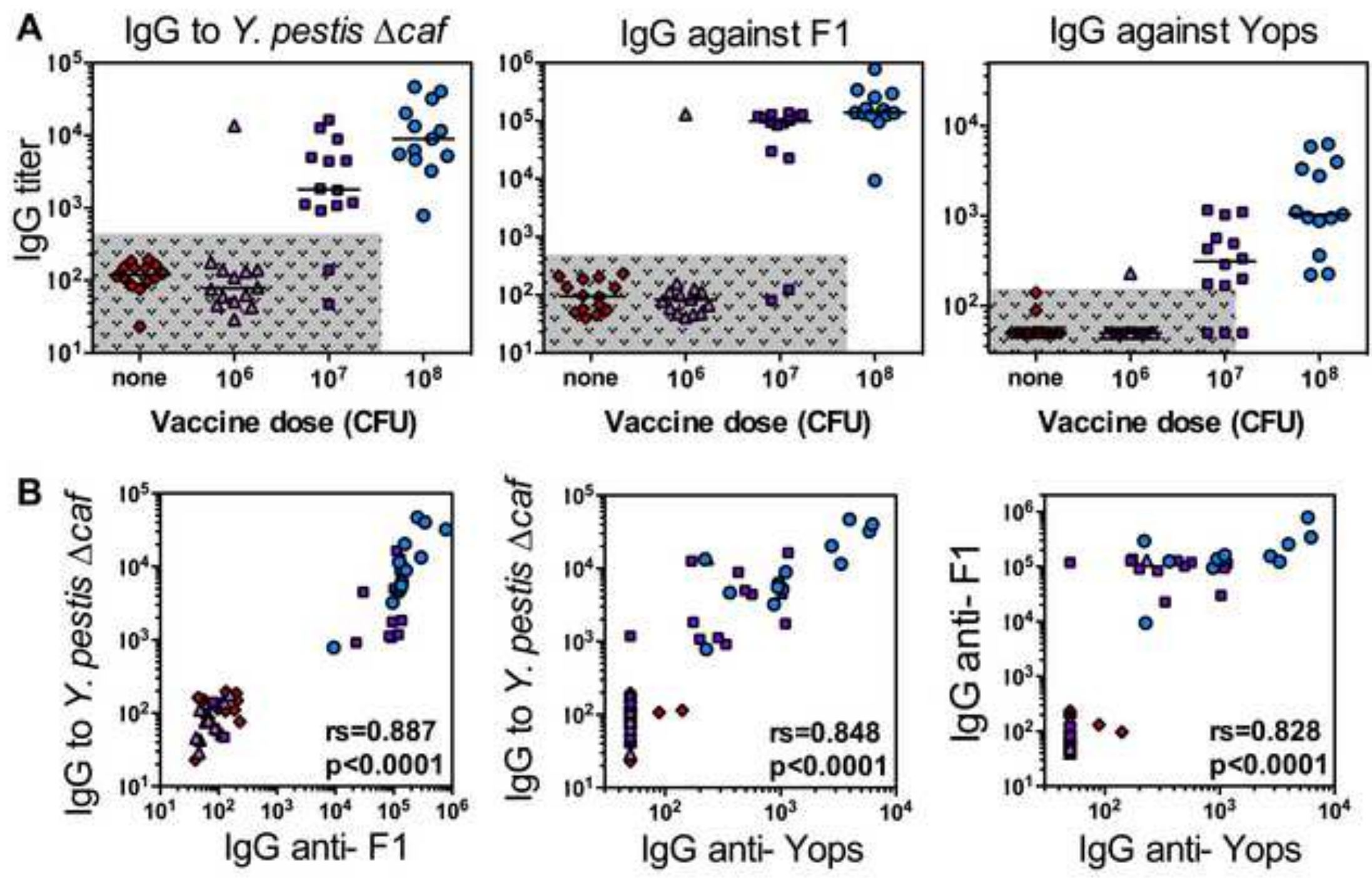


\section{Figure 4}

A

BALB/c mice
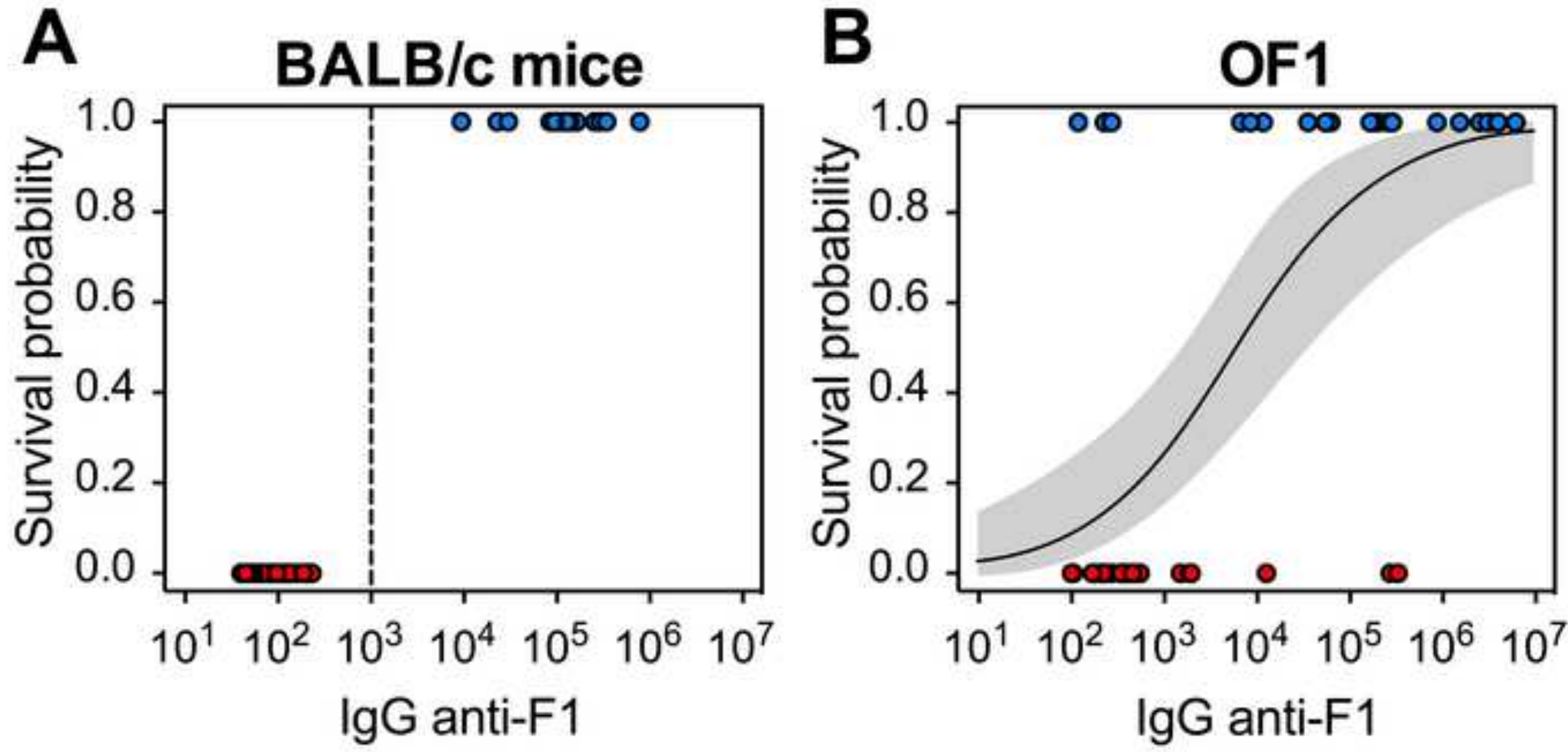


\section{Figure 3}
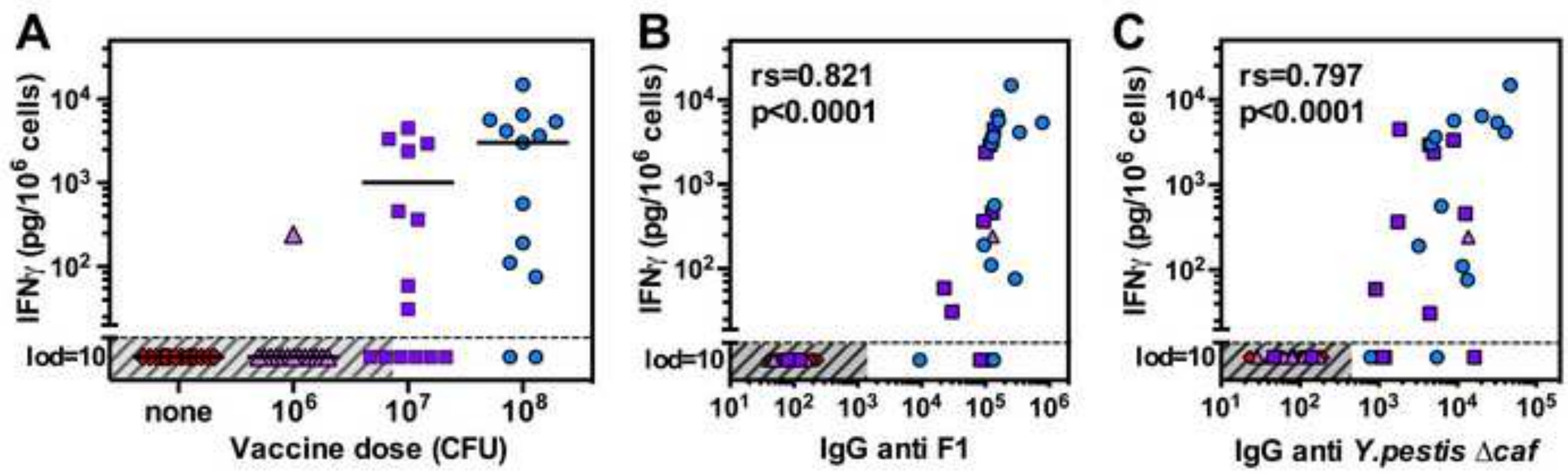